Supporting Information

\title{
Tuning the Range of Polyacrylamide Gel Stiffness for Mechanobiology Applications
}

Aleksandra K. Denisin ${ }^{a, b}$ and Beth L. Pruitt ${ }^{b, c, d^{*}}$

a. Department of Bioengineering, Stanford University, 443 Via Ortega, Shriram Center, Room 119, Stanford, CA 94305-4125, USA.

b. Department of Mechanical Engineering, Stanford University, Building 530

440 Escondido Mall, Stanford, CA 94305, USA.

c. Stanford Cardiovascular Institute, Stanford University, 265 Campus Drive

Stanford, CA 94305, USA.

d. Department of Molecular and Cellular Physiology, Stanford University School of Medicine, 279 Campus Drive, Beckman Center, Room B100A, Stanford, CA 94305-5345

*Corresponding Author: pruitt@stanford.edu 
Figure S1. Surface VP SEM images of various formulations of polyacrylamide gels. The gel structure cannot be resolved given the $50 \mu \mathrm{m}$ limit of the environmental VP SEM. Scale bars show $50 \mu \mathrm{m}$.

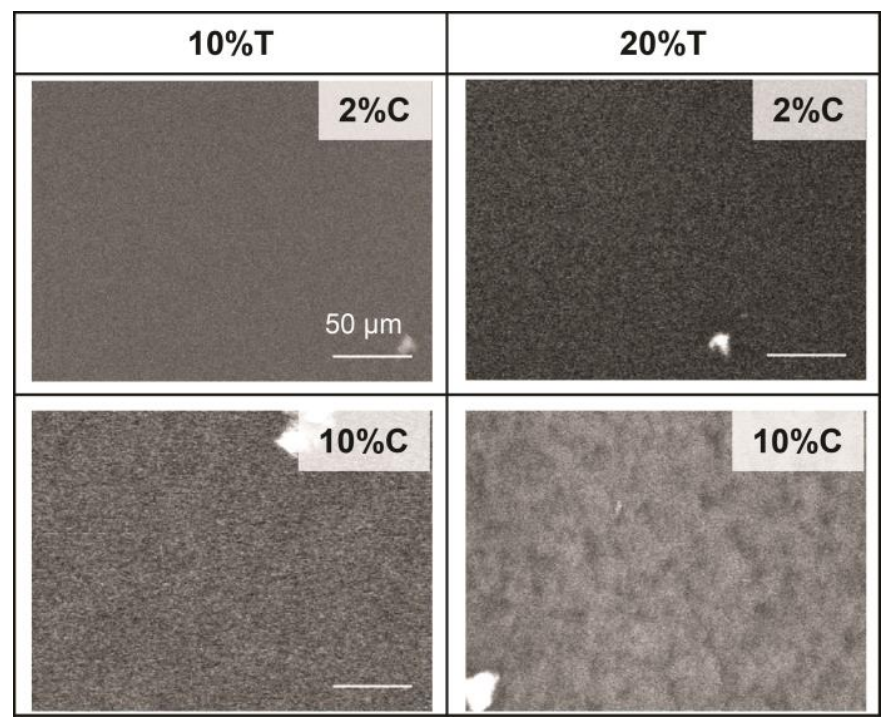


Figure S2. Edge FE SEM images of various formulations of polyacrylamide gels. Across the same polymer content (T), 2\% C samples which followed the power law relationship appear on the top row, $4-8 \% \mathrm{C}$ samples at the inflection point of deviating from the power law appear in the middle row, and $10 \% \mathrm{C}$ samples which are well past the inflection point are in the bottom row. Scale bars show $200 \mathrm{~nm}$.
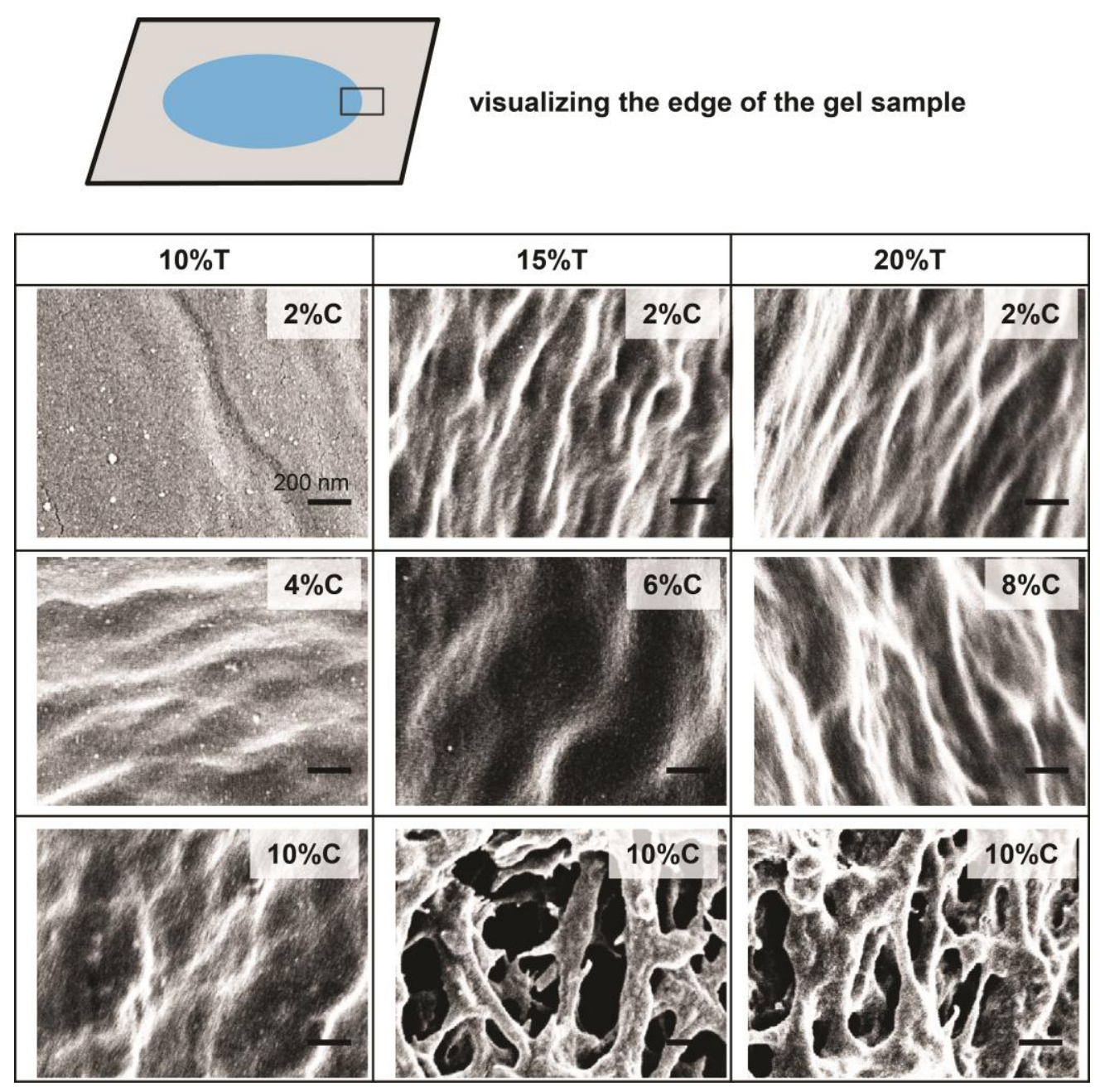
Figure S3. Polymerization time alters swelling ratio. 10\% T 2\% C gels exhibit the greatest range in the swelling ratio when polymerized in 30 minutes at room temperature, compared to polymerization overnight. Additionally, $10 \% \mathrm{~T} 2 \% \mathrm{C}$ gels exhibited the largest range overall when compared to other gel formulations polymerized for both 30 minutes and overnight.

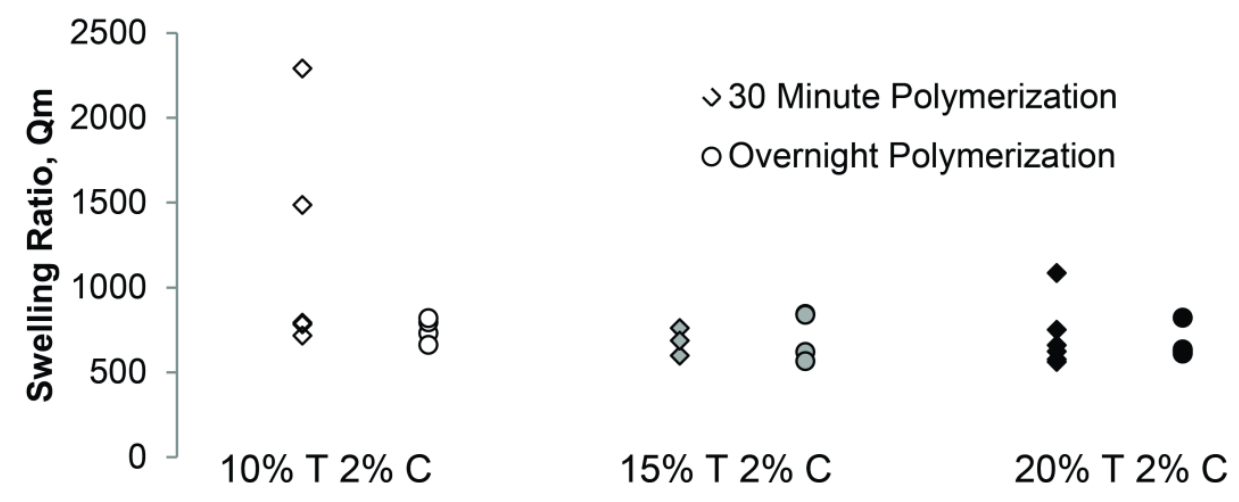

Table S1. Metadata analysis of elastic modulus values

\begin{tabular}{|l|l|l|l|l|l|}
\hline 1st Author & Year & $\begin{array}{l}\text { Reported } \\
\text { E }(\mathbf{k P a})\end{array}$ & Measurement Method & $\begin{array}{l}\mathbf{\% T} \\
\mathbf{( w / v )}\end{array}$ & $\mathbf{\% C}$ \\
\hline Boudou & & & & \\
\hline Boudou & 2006 & 0.61 & Micropipette Aspiration & 2.05 & 2.44 \\
\hline Boudou & 2006 & 1.22 & Micropipette Aspiration & 2.10 & 4.76 \\
\hline Boudou & 2006 & 1.84 & Micropipette Aspiration & 2.15 & 6.98 \\
\hline Boudou & 2006 & 2.45 & Micropipette Aspiration & 2.20 & 9.09 \\
\hline Boudou & 2006 & 0.78 & Micropipette Aspiration & 4.05 & 1.23 \\
\hline Boudou & 2006 & 1.57 & Micropipette Aspiration & 4.10 & 2.44 \\
\hline Boudou & 2006 & 3.13 & Micropipette Aspiration & 4.20 & 4.76 \\
\hline Boudou & 2006 & 3.17 & Micropipette Aspiration & 7.05 & 0.71 \\
\hline
\end{tabular}




\begin{tabular}{|l|l|l|l|l|l|}
\hline Boudou & 2006 & 12.69 & Micropipette Aspiration & 7.20 & 2.78 \\
\hline Boudou & 2006 & 8.16 & Micropipette Aspiration & 9.05 & 0.55 \\
\hline Boudou & 2006 & 16.33 & Micropipette Aspiration & 9.10 & 1.10 \\
\hline Boudou & 2006 & 24.49 & Micropipette Aspiration & 9.15 & 1.64 \\
\hline Boudou & 2006 & 32.65 & Micropipette Aspiration & 9.20 & 2.17 \\
\hline Wang & 2002 & 8.70 & Macroscale Stretch & 2.10 & 4.76 \\
\hline Wang & 2002 & 1.30 & Macroscale Stretch & 2.25 & 11.11 \\
\hline Tse & 2010 & 0.20 & AFM microindentation (Hertz) & 3.03 & 0.99 \\
\hline Tse & 2010 & 0.48 & AFM microindentation (Hertz) & 3.06 & 1.96 \\
\hline Tse & 2010 & 1.10 & AFM microindentation (Hertz) & 3.10 & 3.23 \\
\hline Tse & 2010 & 1.37 & AFM microindentation (Hertz) & 3.15 & 4.76 \\
\hline Tse & 2010 & 1.67 & AFM microindentation (Hertz) & 3.23 & 6.98 \\
\hline Tse & 2010 & 1.78 & AFM microindentation (Hertz) & 3.30 & 9.09 \\
\hline Tse & 2010 & 0.71 & AFM microindentation (Hertz) & 4.03 & 0.74 \\
\hline Tse & 2010 & 1.16 & AFM microindentation (Hertz) & 4.06 & 1.48 \\
\hline Tse & 2010 & 2.01 & AFM microindentation (Hertz) & 4.10 & 2.44 \\
\hline Tse & 2010 & 1.80 & AFM microindentation (Hertz) & 5.06 & 1.19 \\
\hline Tse & 2010 & 2.55 & AFM microindentation (Hertz) & 4.15 & 3.61 \\
\hline Tse & 2010 & 3.13 & AFM microindentation (Hertz) & 4.23 & 5.33 \\
\hline Tse & 2010 & 3.24 & AFM microindentation (Hertz) & 4.30 & 6.98 \\
\hline Tse & 2010 & 8.44 & AFM microindentation (Hertz) & 5.23 & 4.31 \\
\hline Tse & 2010 & AFM microindentation (Hertz) & 5.30 & 5.66 \\
\hline Tse & AFM microindentation (Hertz) & 8.05 & 0.60 \\
\hline Tse & 2010 & AFM microindentation (Hertz) & 5.03 & 0.60 \\
\hline Tse & 2010 & 5.10 & 1.96 \\
\hline Tse & 2010 & 5.15 & 2.91 \\
\hline
\end{tabular}




\begin{tabular}{|l|l|l|l|l|l|}
\hline Tse & 2010 & 19.66 & AFM microindentation (Hertz) & 8.26 & 3.17 \\
\hline Tse & 2010 & 40.40 & AFM microindentation (Hertz) & 8.48 & 5.66 \\
\hline Tse & 2010 & 2.83 & AFM microindentation (Hertz) & 10.03 & 0.30 \\
\hline Tse & 2010 & 7.43 & AFM microindentation (Hertz) & 10.06 & 0.60 \\
\hline Tse & 2010 & 10.61 & AFM microindentation (Hertz) & 10.10 & 0.99 \\
\hline Tse & 2010 & 16.70 & AFM microindentation (Hertz) & 10.15 & 1.48 \\
\hline Tse & 2010 & 23.43 & AFM microindentation (Hertz) & 10.23 & 2.20 \\
\hline Flanagan & 2002 & 0.18 & Fluid Rheometry & 3.04 & 1.32 \\
\hline Flanagan & 2002 & 0.71 & Fluid Rheometry & 3.06 & 1.96 \\
\hline Flanagan & 2002 & 0.83 & Fluid Rheometry & 3.08 & 2.60 \\
\hline Flanagan & 2002 & 1.36 & Fluid Rheometry & 3.12 & 3.85 \\
\hline Flanagan & 2002 & 1.63 & Fluid Rheometry & 3.20 & 6.25 \\
\hline Flanagan & 2002 & 1.63 & Fluid Rheometry & 3.50 & 14.29 \\
\hline Trappmann & 2012 & 0.50 & Microindentation & 5.01 & 0.20 \\
\hline Trappmann & 2012 & 2.00 & Microindentation & 5.02 & 0.40 \\
\hline Trappmann & 2012 & 21.50 & Microindentation & 8.15 & 1.84 \\
\hline Trappmann & 2012 & 115.00 & Microindentation & 20.38 & 1.84 \\
\hline Trappmann & 2012 & 231.80 & Microindentation & 43.00 & 6.98 \\
\hline Trappmann & 2012 & 744.40 & Microindentation & 5.03 & 0.50 \\
\hline Engler (A) & 2004 & 1.00 & AFM microindentation (Hertz) & 5.06 & 1.19 \\
\hline Engler (A) & 2004 & 2.00 & AFM microindentation (Hertz) & 5.10 & 1.96 \\
\hline Engler (A) & 2004 & 3.00 & AFM microindentation (Hertz) & 5.15 & 2.91 \\
\hline Engler (A) & 2004 & 5.80 & AFM microindentation (Hertz) & 5.23 & 4.31 \\
\hline Engler (A) & 2004 & 7.00 & AFM microindentation (Hertz) & 5.30 & 5.66 \\
\hline Engler (A) & 2004 & 8.00 & AFM microindentation (Hertz) & 0.50 \\
\hline Engler (A) & 2004 & 1.50 & Macroscale Stretch & 5.03 \\
\hline
\end{tabular}




\begin{tabular}{|c|c|c|c|c|c|}
\hline Engler (A) & 2004 & 2.80 & Macroscale Stretch & 5.06 & 1.19 \\
\hline Engler (A) & 2004 & 3.80 & Macroscale Stretch & 5.10 & 1.96 \\
\hline Engler (A) & 2004 & 6.50 & Macroscale Stretch & 5.15 & 2.91 \\
\hline Engler (A) & 2004 & 8.00 & Macroscale Stretch & 5.23 & 4.31 \\
\hline Engler (A) & 2004 & 8.25 & Macroscale Stretch & 5.30 & 5.66 \\
\hline Engler $(\mathrm{B})^{7}$ & 2004 & 1.40 & AFM microindentation (Hertz) & 5.03 & 0.60 \\
\hline Engler (B) & 2004 & 8.10 & AFM microindentation (Hertz) & 5.30 & 5.66 \\
\hline Munevar $^{8}$ & 2001 & 28.00 & AFM microindentation (Hertz) & 5.10 & 1.96 \\
\hline Pelham $^{9}$ & 1997 & 15.00 & Microindentation & 10.03 & 0.30 \\
\hline Pelham & 1997 & 25.00 & Macroscale Stretch & 10.07 & 0.70 \\
\hline Pelham & 1997 & 35.00 & Macroscale Stretch & 10.13 & 1.28 \\
\hline Pelham & 1997 & 68.00 & Macroscale Stretch & 10.26 & 2.53 \\
\hline Hazeltine $^{10}$ & 2012 & 5.00 & Microindentation & 10.25 & 2.44 \\
\hline Hazeltine & 2012 & 20.00 & Microindentation & 10.10 & 0.99 \\
\hline Hazeltine & 2012 & 50.00 & Microindentation & 10.30 & 2.91 \\
\hline Hazeltine & 2012 & 60.00 & Microindentation & 10.40 & 3.85 \\
\hline Hazeltine & 2012 & 70.00 & Microindentation & 10.50 & 4.76 \\
\hline Hazeltine & 2012 & 100.00 & Microindentation & 10.60 & 5.66 \\
\hline Dembo $^{11}$ & 1999 & 6.20 & Macroscale Compression & 10.03 & 0.30 \\
\hline Dembo & 1999 & 100.00 & Macroscale Compression & 10.24 & 2.34 \\
\hline Mahaffy $^{12}$ & 2000 & 2.16 & AFM microindentation & 4.11 & 2.60 \\
\hline Geissler $^{13}$ & 1981 & 0.28 & Dynamic Light Scattering & 1.82 & 42.86 \\
\hline Geissler & 1981 & 0.56 & Dynamic Light Scattering & 1.98 & 27.27 \\
\hline Geissler & 1981 & 0.74 & Dynamic Light Scattering & 2.20 & 27.27 \\
\hline Geissler & 1981 & 0.25 & Dynamic Light Scattering & 4.74 & 42.86 \\
\hline
\end{tabular}




\begin{tabular}{|c|c|c|c|c|c|}
\hline Geissler & 1981 & 0.38 & Dynamic Light Scattering & 5.74 & 42.86 \\
\hline Geissler & 1981 & 0.35 & Dynamic Light Scattering & 6.00 & 42.86 \\
\hline Geissler & 1981 & 0.60 & Dynamic Light Scattering & 6.04 & 27.27 \\
\hline Geissler & 1981 & 0.68 & Dynamic Light Scattering & 6.45 & 27.27 \\
\hline Geissler & 1981 & 0.89 & Dynamic Light Scattering & 6.61 & 27.27 \\
\hline Simmons $^{14}$ & 2012 & 5.90 & AFM microindentation (Oliver Pharr) & 10.05 & 0.50 \\
\hline Simmons & 2012 & 20.10 & AFM microindentation (Oliver Pharr) & 10.50 & 4.76 \\
\hline Sazonova $^{15}$ & 2011 & 25.00 & AFM microindentation (Hertz) & 10.10 & 0.99 \\
\hline Sazonova & 2011 & 70.00 & AFM microindentation (Hertz) & 10.40 & 3.85 \\
\hline Sazonova & 2011 & 136.00 & AFM microindentation (Hertz) & 12.60 & 4.76 \\
\hline Sazonova & 2011 & 22.00 & Microindentation & 10.10 & 0.99 \\
\hline Sazonova & 2011 & 68.00 & Microindentation & 10.40 & 3.85 \\
\hline Sazonova & 2011 & 130.00 & Microindentation & 12.60 & 4.76 \\
\hline Markert $^{16}$ & 2013 & 1.30 & AFM microindentation (Hertz) & 5.17 & 3.33 \\
\hline Markert & 2013 & 2.20 & AFM microindentation (Hertz) & 10.34 & 3.33 \\
\hline $\mathrm{Li}^{17}$ & 2011 & 100.00 & AFM microindentation (Hertz) & 20.50 & 2.44 \\
\hline $\mathrm{Li}$ & 2011 & 300.00 & AFM microindentation (Hertz) & 21.50 & 6.98 \\
\hline $\mathrm{Li}$ & 2011 & 300.00 & AFM microindentation (Hertz) & 22.00 & 9.09 \\
\hline $\mathrm{Li}$ & 2011 & 500.00 & AFM microindentation (Hertz) & 22.50 & 11.11 \\
\hline $\mathrm{Li}$ & 2011 & 200.00 & AFM microindentation (Hertz) & 31.00 & 3.23 \\
\hline $\mathrm{Li}$ & 2011 & 500.00 & AFM microindentation (Hertz) & 32.00 & 6.25 \\
\hline $\mathrm{Li}$ & 2011 & 1100.00 & AFM microindentation (Hertz) & 33.00 & 9.09 \\
\hline $\mathrm{Li}$ & 2011 & 300.00 & Macroscale Compression & 20.50 & 2.44 \\
\hline $\mathrm{Li}$ & 2011 & 500.00 & Macroscale Compression & 21.50 & 6.98 \\
\hline $\mathrm{Li}$ & 2011 & 800.00 & Macroscale Compression & 22.00 & 9.09 \\
\hline $\mathrm{Li}$ & 2011 & 500.00 & Macroscale Compression & 31.00 & 3.23 \\
\hline
\end{tabular}




\begin{tabular}{|c|c|c|c|c|c|}
\hline $\mathrm{Li}$ & 2011 & 1200.00 & Macroscale Compression & 32.00 & 6.25 \\
\hline $\mathrm{Li}$ & 2011 & 1400.00 & Macroscale Compression & 33.00 & 9.09 \\
\hline Galli $^{18}$ & 2009 & 370.00 & AFM microindentation & 21.05 & 5.00 \\
\hline Galli & 2009 & 370.00 & AFM microindentation & 21.05 & 5.00 \\
\hline Galli & 2009 & 350.00 & AFM microindentation & 21.05 & 5.00 \\
\hline Galli & 2009 & 500.00 & AFM microindentation & 21.05 & 5.00 \\
\hline $\mathrm{Jacot}^{19}$ & 2006 & 5.00 & AFM microindentation (Oliver Pharr) & 8.03 & 0.31 \\
\hline Jacot & 2006 & 10.00 & AFM microindentation (Oliver Pharr) & 8.05 & 0.62 \\
\hline Jacot & 2006 & 15.00 & AFM microindentation (Oliver Pharr) & 8.10 & 1.23 \\
\hline Jacot & 2006 & 22.00 & AFM microindentation (Oliver Pharr) & 8.15 & 1.84 \\
\hline Jacot & 2006 & 90.00 & AFM microindentation (Oliver Pharr) & 10.50 & 4.76 \\
\hline Ling $^{20}$ & 2010 & 4.40 & AFM microindentation (Oliver Pharr) & 5.00 & 0.00 \\
\hline Ling & 2010 & 9.69 & AFM microindentation (Oliver Pharr) & 6.00 & 0.00 \\
\hline $\mathrm{Wen}^{21}$ & 2014 & 4.50 & AFM microindentation (Hertz) & 4.40 & 9.09 \\
\hline Wen & 2014 & 4.80 & AFM microindentation (Hertz) & 6.06 & 0.99 \\
\hline Wen & 2014 & 4.10 & AFM microindentation (Hertz) & 10.02 & 0.20 \\
\hline Wen & 2014 & 13.90 & AFM microindentation (Hertz) & 6.45 & 6.98 \\
\hline Wen & 2014 & 13.80 & AFM microindentation (Hertz) & 10.10 & 0.99 \\
\hline Wen & 2014 & 14.20 & AFM microindentation (Hertz) & 20.03 & 0.15 \\
\hline Wen & 2014 & 27.60 & AFM microindentation (Hertz) & 8.55 & 6.43 \\
\hline Wen & 2014 & 32.00 & AFM microindentation (Hertz) & 10.30 & 2.91 \\
\hline Wen & 2014 & 30.70 & AFM microindentation (Hertz) & 20.18 & 0.89 \\
\hline Fereol $^{22}$ & 2006 & 40.00 & Microindentation & 10.07 & 0.70 \\
\hline Fereol & 2006 & 160.00 & Microindentation & 10.30 & 2.91 \\
\hline Ambrosi $^{23}$ & 2009 & 10.70 & Fluid Rheometry & 10.03 & 0.32 \\
\hline
\end{tabular}




\begin{tabular}{|l|l|l|l|l|l|}
\hline Ambrosi & 2009 & 15.09 & Macroscale Indentation & 10.07 & 0.71 \\
\hline Ambrosi & 2009 & 24.27 & Macroscale Indentation & 10.15 & 1.50 \\
\hline Ambrosi & 2009 & 41.82 & Macroscale Indentation & 10.30 & 2.93 \\
\hline Yeung ${ }^{4}$ & 2005 & 49.26 & Fluid Rheometry & 12.15 & 1.23 \\
\hline Yeung & 2005 & 90.00 & Fluid Rheometry & 12.28 & 2.30 \\
\hline Yeung & 2005 & 107.40 & Fluid Rheometry & 12.45 & 3.61 \\
\hline Yeung & 2005 & 123.75 & Fluid Rheometry & 12.56 & 4.44 \\
\hline Yeung & 2005 & 158.55 & Fluid Rheometry & 12.59 & 4.65 \\
\hline Yeung & 2005 & 26.04 & Fluid Rheometry & 7.81 & 3.93 \\
\hline Yeung & 2005 & 15.30 & Fluid Rheometry & 7.71 & 2.66 \\
\hline Yeung & 2005 & 11.53 & Fluid Rheometry & 7.65 & 1.97 \\
\hline Yeung & 2005 & 8.38 & Fluid Rheometry & 7.60 & 1.35 \\
\hline Yeung & 2005 & 6.78 & Fluid Rheometry & 7.58 & 1.01 \\
\hline Yeung & 2005 & 4.43 & Fluid Rheometry & 7.55 & 0.66 \\
\hline Yeung & 2005 & 2.03 & Fluid Rheometry & 7.53 & 0.40 \\
\hline Yeung & 2005 & 0.65 & Fluid Rheometry & 7.51 & 0.13 \\
\hline Yeung & 2005 & 5.84 & Fluid Rheometry & 5.70 & 3.54 \\
\hline Yeung & 2005 & 4.73 & Fluid Rheometry & 5.65 & 2.71 \\
\hline Yeung & 2005 & 4.27 & Fluid Rheometry & 5.60 & 1.82 \\
\hline Yeung & 2005 & 1.71 & Fluid Rheometry & 3.58 & 1.36 \\
\hline Yeung & 2005 & 1.25 & Fluid Rheometry & 3.55 & 0.90 \\
\hline Yeung & 2005 & 1.46 & Fluid Rheometry & 3.20 & 14.31 \\
\hline Yeung & 2005 & 1.47 & Fluid Rheometry & 6.31 \\
\hline Yeung & 0.66 & Fluid Rheometry & 3.85 \\
\hline Yeung & Fluid Rheometry & Fluid Rheometry & 2.67 \\
\hline Yeung & 2005 & & 3.33 & $5-10$ \\
\hline
\end{tabular}




\begin{tabular}{|l|l|l|l|l|l|}
\hline Yeung & 2005 & 0.15 & Fluid Rheometry & 3.04 & 1.33 \\
\hline Damljanovic ${ }^{25}$ & 2005 & 17.39 & Microindentation & 4.57 & 1.53 \\
\hline Damljanovic & 2005 & 20.88 & Microindentation & 4.60 & 2.17 \\
\hline Damljanovic & 2005 & 35.28 & Microindentation & 4.65 & 3.23 \\
\hline Damljanovic & 2005 & 26.72 & Microindentation & 5.07 & 1.34 \\
\hline Damljanovic & 2005 & 33.91 & Microindentation & 5.10 & 1.96 \\
\hline Damljanovic & 2005 & 54.10 & Microindentation & 5.15 & 2.91 \\
\hline Damljanovic & 2005 & 35.24 & Microindentation & 6.07 & 1.15 \\
\hline Damljanovic & 2005 & 48.07 & Microindentation & 6.10 & 1.64 \\
\hline Damljanovic & 2005 & 80.33 & Microindentation & 6.15 & 2.44 \\
\hline Damljanovic & 2005 & 61.00 & Microindentation & 8.07 & 0.87 \\
\hline Damljanovic & 2005 & 80.73 & Microindentation & 8.10 & 1.23 \\
\hline Damljanovic & 2005 & 123.00 & Microindentation & 8.15 & 1.84 \\
\hline
\end{tabular}


Table S2. Parameters used to measure force-distance curves

\begin{tabular}{|l|l|l|l|}
\hline \multicolumn{2}{|l|}{ Formulation } & \multicolumn{2}{l|}{} \\
\hline$\% \mathrm{~T}$ & $\% \mathrm{C}$ & Pull $(\mu \mathrm{m})$ & Push $(\mu \mathrm{m})$ \\
\hline 10 & $0.2-1$ & 10 & 20 \\
\hline 10 & $2-3$ & 8 & 6 \\
\hline 10 & $4-8$ & 8 & 5 \\
\hline 15 & 1 & 5 & 4 \\
\hline 15 & 2 & 5 & 3 \\
\hline 15 & $4-10$ & 5 & 2 \\
\hline 20 & 0.2 & 7 & 5 \\
\hline 20 & $1-2$ & 5 & 2 \\
\hline 20 & 4 & 5 & 2 \\
\hline 20 & 6 & 5 & 2 \\
\hline 20 & 7.8 & 5 & 1.5 \\
\hline
\end{tabular}


Table S3. Analysis of polyacrylamide gel elastic modulus during aging

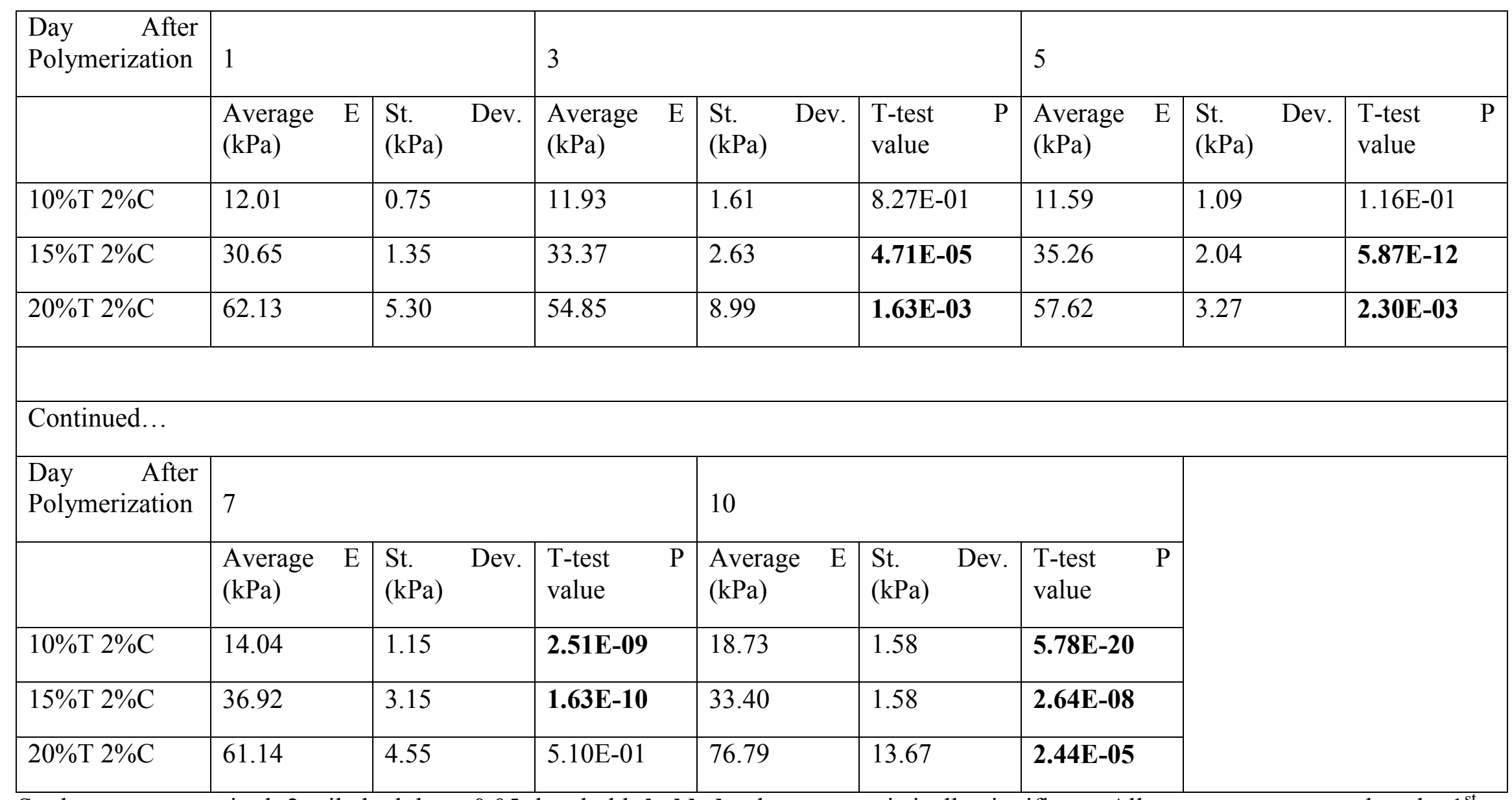

Student t-test, unpaired, 2 tailed. alpha $=0.05$ threshold, bolded values are statistically significant. All t-tests are compared to the $1^{\text {st }}$ day after polymerization. 
Table S4. Hydrogel Formulations and Polymerization Conditions

\begin{tabular}{|c|c|c|c|c|c|c|c|}
\hline $\begin{array}{l}\text { Expected } \\
\text { Elastic } \\
\text { Modulus } \\
(\mathbf{k P a})\end{array}$ & $\% \mathrm{~T}$ & $\% \mathrm{C}$ & $\begin{array}{l}\text { milliQ } \\
\text { water }(\mu \mathrm{l})\end{array}$ & $\begin{array}{l}\text { Acrylamide } \\
(\mu \mathrm{l} \text { of } 0.5 \\
\mathrm{mg} / \mathrm{ml} \\
\text { stock) }\end{array}$ & $\begin{array}{l}\text { Bis- } \\
\text { acrylamide } \\
\text { ( } \mu \text { l of } 0.025 \\
\mathrm{mg} / \mathrm{ml} \\
\text { stock) }\end{array}$ & $\begin{array}{l}\text { APS }(\mu \mathrm{l}) \\
\text { of } 10 \% \\
\text { solution) }\end{array}$ & $\begin{array}{l}\text { TEMED } \\
(\mu \mathrm{l})\end{array}$ \\
\hline 6.29 & 10 & 1 & 756.50 & 198 & 40 & 5 & 0.50 \\
\hline 14.04 & 10 & 2 & 718.50 & 196 & 80 & 5 & 0.50 \\
\hline 13.79 & 10 & 3 & 680.50 & 194 & 120 & 5 & 0.50 \\
\hline 19.61 & 10 & 4 & 644.50 & 190 & 160 & 5 & 0.50 \\
\hline 32.29 & 15 & 1 & 636.50 & 297 & 61 & 5 & 0.50 \\
\hline 38.33 & 15 & 2 & 579.50 & 294 & 121 & 5 & 0.50 \\
\hline 69.75 & 15 & 4 & 464.50 & 289 & 241 & 5 & 0.50 \\
\hline 93.46 & 15 & 6 & 352.50 & 282 & 360 & 5 & 0.50 \\
\hline 10.14 & 20 & 0.2 & 578.50 & 400 & 16 & 5 & 0.50 \\
\hline 27.25 & 20 & 1 & 517.50 & 397 & 80 & 5 & 0.50 \\
\hline 51.41 & 20 & 2 & 441.50 & 392 & 161 & 5 & 0.50 \\
\hline 99.25 & 20 & 3 & 441.50 & 392 & 161 & 5 & 0.50 \\
\hline 142.91 & 20 & 6 & 136.50 & 377 & 481 & 5 & 0.50 \\
\hline 163.77 & 20 & 7.8 & 0.50 & 369 & 625 & 5 & 0.50 \\
\hline
\end{tabular}

All gels were tested within 24 hours of polymerization and were stored at room temperature. The elastic modulus noted is the average of 25 microscale indentations fit to the Hertz model for the first $2 \mu \mathrm{m}$ of indentation into the substrate. We are showing gels which responded via a power law relation of increasing elastic modulus when crosslinker content was increased and thus we are excluding gels beyond the inflection point which are heterogeneous and contain inhomogeneities. The total volume of each solution was 1 milliliter of gel precursor. 
(1) Boudou, T.; Ohayon, J.; Picart, C.; Tracqui, P. An extended relationship for the characterization of Young's modulus and Poisson's ratio of tunable polyacrylamide gels. Biorheology 2006, 43, 721-728.

(2) Wang, N.; Tolic-Norrelykke, I. M.; Chen, J.; Mijailovich, S. M.; Butler, J. P.; Fredberg, J. J.; Stamenovic, D. Cell prestress. I. Stiffness and prestress are closely associated in adherent contractile cells. Am. J. Physiol. Cell. Physiol. 2002, 282, C606-16, DOI: 10.1152/ajpcell.00269.2001 [doi].

(3) Tse, J. R.; Engler, A. J. Preparation of hydrogel substrates with tunable mechanical properties. Curr. Protoc. Cell Biol. 2010, Supplement 47, 10.16. 1-10.16. 16.

(4) Flanagan, L. A.; Ju, Y.; Marg, B.; Osterfield, M.; Janmey, P. A. Neurite branching on deformable substrates. Neuroreport 2002, 13, 2411.

(5) Trappmann, B.; Gautrot, J. E.; Connelly, J. T.; Strange, D. G. T.; Li, Y.; Oyen, M. L.; Stuart, M. A. C.; Boehm, H.; Li, B.; Vogel, V. Extracellular-matrix tethering regulates stem-cell fate. Nature Mat. 2012, 11, 642-649.

(6) Engler, A.; Bacakova, L.; Newman, C.; Hategan, A.; Griffin, M.; Discher, D. Substrate compliance versus ligand density in cell on gel responses. Biophys. J. 2004, 86, 617-628.

(7) Engler, A. J.; Richert, L.; Wong, J. Y.; Picart, C.; Discher, D. E. Surface probe measurements of the elasticity of sectioned tissue, thin gels and polyelectrolyte multilayer films: correlations between substrate stiffness and cell adhesion. Surf. Sci. 2004, 570, 142-154.

(8) Munevar, S.; Wang, Y.; Dembo, M. Traction force microscopy of migrating normal and Hras transformed $3 \mathrm{~T} 3$ fibroblasts. Biophys. J. 2001, 80, 1744-1757. 
(9) Pelham, R. J.; Wang, Y. Cell locomotion and focal adhesions are regulated by substrate flexibility. Proc. Natl. Acad. Sci. 1997, 94, 13661-13665.

(10) Hazeltine, L. B.; Simmons, C. S.; Salick, M. R.; Lian, X.; Badur, M. G.; Han, W.; Delgado, S. M.; Wakatsuki, T.; Crone, W. C.; Pruitt, B. L. Effects of substrate mechanics on contractility of cardiomyocytes generated from human pluripotent stem cells. International journal of cell biology 2012, 2012.

(11) Dembo, M.; Wang, Y. Stresses at the cell-to-substrate interface during locomotion of fibroblasts. Biophys. J. 1999, 76, 2307-2316.

(12) Mahaffy, R.; Shih, C.; MacKintosh, F.; Käs, J. Scanning probe-based frequencydependent microrheology of polymer gels and biological cells. Phys. Rev. Lett. 2000, 85, 880.

(13) Geissler, E.; Hecht, A. The Poisson ratio in polymer gels. Macromolecules 1980, 13, 1276-1280.

(14) Simmons, C. S.; Ribeiro, A.; Pruitt, B. L. Formation of composite polyacrylamide and silicone substrates for independent control of stiffness and strain. Lab on a Chip 2012.

(15) Sazonova, O. V.; Lee, K. L.; Isenberg, B. C.; Rich, C. B.; Nugent, M. A.; Wong, J. Y. Cell-cell interactions mediate the response of vascular smooth muscle cells to substrate stiffness. Biophys. J. 2011, 101, 622-630.

(16) Markert, C. D.; Guo, X.; Skardal, A.; Wang, Z.; Bharadwaj, S.; Zhang, Y.; Bonin, K.; Guthold, M. Characterizing the micro-scale elastic modulus of hydrogels for use in regenerative medicine. J. Mech. Behav. Biomed. Mater. 2013, 27, 115-127. 
(17) Li, C.; Allen, J.; Alliston, T.; Pruitt, L. A. The use of polyacrylamide gels for mechanical calibration of cartilage-A combined nanoindentation and unconfined compression study. Journal of the mechanical behavior of biomedical materials 2011, 4, 1540-1547.

(18) Galli, M.; Comley, K. S.; Shean, T. A.; Oyen, M. L. Viscoelastic and poroelastic mechanical characterization of hydrated gels. J. Mater. Res. 2009, 24, 973-979.

(19) Jacot, J. G.; Dianis, S.; Schnall, J.; Wong, J. Y. A simple microindentation technique for mapping the microscale compliance of soft hydrated materials and tissues. Journal of Biomedical Materials Research Part A 2006, 79, 485-494.

(20) Ling, T.; Jin, Q.; Yao, H.; Zheng, H. Design and Characterization of a Tissue-Mimicking Phantom for Ultrasonic Elastography. Bioinformatics and Biomedical Engineering (iCBBE), 2010 4th International Conference on. 2010, 1-4.

(21) Wen, J. H.; Vincent, L. G.; Fuhrmann, A.; Choi, Y. S.; Hribar, K. C.; Taylor-Weiner, H.; Chen, S.; Engler, A. J. Interplay of matrix stiffness and protein tethering in stem cell differentiation. Nature Mat. 2014, 13, 979-987.

(22) Féréol, S.; Fodil, R.; Labat, B.; Galiacy, S.; Laurent, V. M.; Louis, B.; Isabey, D.; Planus, E. Sensitivity of alveolar macrophages to substrate mechanical and adhesive properties. Cell Motil. Cytoskeleton 2006, 63, 321-340.

(23) Ambrosi, D.; Duperray, A.; Peschetola, V.; Verdier, C. Traction patterns of tumor cells. $J$. Math. Biol. 2009, 58, 163-181. 
(24) Yeung, T.; Georges, P. C.; Flanagan, L. A.; Marg, B.; Ortiz, M.; Funaki, M.; Zahir, N.; Ming, W.; Weaver, V.; Janmey, P. A. Effects of substrate stiffness on cell morphology, cytoskeletal structure, and adhesion. Cell Motil. Cytoskeleton 2005, 60, 24-34.

(25) Damljanovic, V.; Lagerholm, B. C.; Jacobson, K. Bulk and micropatterned conjugation of extracellular matrix proteins to characterized polyacrylamide substrates for cell mechanotransduction assays. BioTechniques 2005, 39, 847. 